\title{
Malaria case clinical profiles and Plasmodium falciparum parasite genetic diversity: a cross sectional survey at two sites of different malaria transmission intensities in Rwanda
}

Fredrick Kateera ${ }^{1,2 *}$, Sam L. Nsobya ${ }^{3,4}$, Stephen Tukwasibwe ${ }^{3}$, Petra F. Mens ${ }^{2,5}$, Emmanuel Hakizimana ${ }^{1}$, Martin P. Grobusch², Leon Mutesa ${ }^{6}$, Nirbhay Kumar ${ }^{7}$ and Michele van Vugt ${ }^{2}$

\begin{abstract}
Background: Malaria remains a public health challenge in sub-Saharan Africa with Plasmodium falciparum being the principal cause of malaria disease morbidity and mortality. Plasmodium falciparum virulence is attributed, in part, to its population-level genetic diversity - a characteristic that has yet to be studied in Rwanda. Characterizing P. falciparum molecular epidemiology in an area is needed for a better understand of malaria transmission and to inform choice of malaria control strategies.

Methods: In this health-facility based survey, malaria case clinical profiles and parasite densities as well as parasite genetic diversity were compared among $P$. falciparum-infected patients identified at two sites of different malaria transmission intensities in Rwanda. Data on demographics and clinical features and finger-prick blood samples for microscopy and parasite genotyping were collected Nested PCR was used to genotype msp-2 alleles of FC27 and 3D7.

Results: Patients' variables of age group, sex, fever (both by patient report and by measured tympanic temperatures), parasite density, and bed net use were found differentially distributed between the higher endemic (Ruhuha) and lower endemic (Mubuga) sites. Overall multiplicity of P. falciparum infection (MOI) was 1.73 but with mean MOI found to vary significantly between 2.13 at Ruhuha and 1.29 at Mubuga $(p<0.0001)$. At Ruhuha, expected heterozygosity $\left(E_{H}\right)$ for FC27 and 3D7 alleles were 0.62 and 0.49, respectively, whilst at Mubuga, $E_{H}$ for FC27 and 3D7 were 0.26 and 0.28 , respectively.
\end{abstract}

Conclusions: In this study, a higher geometrical mean parasite counts, more polyclonal infections, higher $\mathrm{MOI}$, and higher allelic frequency were observed at the higher malaria-endemic (Ruhuha) compared to the lower malariaendemic (Mubuga) area. These differences in malaria risk and $\mathrm{MOI}$ should be considered when choosing setting-specific malaria control strategies, assessing $p$. falciparum associated parameters such as drug resistance, immunity and impact of used interventions, and in proper interpretation of malaria vaccine studies.

Keywords: Malaria, Plasmodium falciparum, Parasite density, Multiplicity of infection, Rwanda

*Correspondence: fkkateera@yahoo.com

${ }^{1}$ Medical Research Centre Division, Rwanda Biomedical Centre,

PO Box 7162, Kigali, Rwanda

Full list of author information is available at the end of the article provided you give appropriate credit to the original author(s) and the source, provide a link to the Creative Commons license, and indicate if changes were made. The Creative Commons Public Domain Dedication waiver (http://creativecommons.org/ publicdomain/zero/1.0/) applies to the data made available in this article, unless otherwise stated. 


\section{Background}

In spite of the significant decline in malaria cases and deaths being reported globally, malaria still accounted for about 200 million cases and over 500,000 deaths in 2014 [1]. The malaria burden decline, particularly in sub-Saharan Africa, has been associated with the rapid scaling-up of interventions including long-lasting insecticide-treated nets (LLINs), indoor residual spraying (IRS) with insecticides, and use of artemisinin-based combinational therapy (ACT) for managing uncomplicated malaria cases [2]. Scale-up of LLINs, IRS and ACT implementation in Rwanda was associated with a more than $50 \%$ decline in malaria morbidity and mortality among children aged under 5 years between 2005 and 2010 [3]. In spite of these declines however, malaria is still a public health challenge with the entire Rwandese population considered as being at risk.

Human malaria infections exhibit a broad clinical spectrum ranging from asymptomatic infection to severe life-threatening disease. Disease severity is influenced by interactions between parasite, human host and environmental factors, including, but not limited to, antimalaria therapies used, levels of immunity, age, sex, and pregnancy status [4]. With regard to anti-malaria therapies in Rwanda, resistance in Plasmodium falciparum in the past led to chloroquine being replaced with amodiaquine (AQ) + sulfadoxine-pyrimethamine (SP) in 2001 and then later, $\mathrm{AQ}+\mathrm{SP}$ combination was subsequently replaced with artemether-lumefantrine (AL) in 2006, as first-line anti-malarial therapies for uncomplicated malaria. Malaria transmission levels and the associated risk of morbidity and mortality show a spatial heterogeneity even within small countries such as Rwanda $[5,6]$. Current Rwandan malaria heterogeneity is partly influenced by the variations in type and intensity of malaria control interventions deployed across different settings as well as the baseline residual transmission potentials at the four different malaria transmission zones [5]. Understanding malaria disease severity, including clinical features and parasitaemia levels associated with malaria disease, in populations from areas of differing malaria transmission intensities is needed for decision making on which control tools may have optimal impact.

Plasmodium falciparum is the most prevalent cause of malaria morbidity and mortality in Rwanda [5]. Plasmodium falciparum virulence is mediated, in part, by its population-level genetic diversity which has been reported to influence malaria disease pathology [7-9], acquisition of immunity $[10,11]$, infection transmission intensity [12-14], and vaccine responses [15, 16]. High malaria-endemic areas tend to have extensive $P$. falciparum genetic diversity with infected humans often found with multiple genotypes. Conversely, low transmission areas tend to yield limited $P$. falciparum genetic diversity with a higher proportion of infections being monoclonal [17-20].

Studying plasmodial molecular epidemiology is essential to understanding malaria transmission. Currently, malaria disease severity among health facility-identified cases as well as population-level parasite diversity remains unknown in Rwanda. This study compared clinical profiles of malaria-confirmed cases, parasite densities and $P$. falciparum genetic diversity $[21,22]$ based on the $m s p-2$ gene-a valid, reliable and highly discriminatory and polymorphic marker used for genetic finger printing, at two sites of differing malaria transmission intensities in Rwanda.

\section{Methods}

\section{Study design and sites}

Rwanda is divided into four malaria ecologic zones based on altitude, climate, level of transmission, and disease vector prevalence [5]. Malaria cases for this cross-sectional survey were recruited from rural Ruhuha sector (Bugesera District, Eastern Province) and Mubuga sector (Karongi District, Western Province) (Fig. 1) located in the highest and lowest malaria transmission zones, respectively [5].

\section{Baseline demographics, clinical features and blood sample collection}

All health facility-visiting cases aged $\geq 6$ months who were microscopically confirmed by the health facility laboratory technicians to be $P$. falciparum infected in the period 15th January to 15th February 2015 were eligible for enrolment. Upon provision of written informed consent, a brief structured questionnaire was administered to collect data on demographics (sex, age, area of residence), fever history, and bed net use on the night before the survey. In addition, body temperature was measured using an electronic tympanic thermometer and a second round of finger-prick blood samples taken off to prepare thick and thin smears for analysis by the study laboratory technicians and for blotting on to filter papers (Whatman 3MM) for use in performing molecular studies.

\section{Preparation of blood films, microscopic examination and quality assurance}

Thick blood smears were stained with $3 \%$ Giemsa for $60 \mathrm{~min}$ and slides read by two blinded study microscopists. In case of two discordant results, a third reader was used to resolve the discrepancy. Using the thick blood smear, parasite densities were enumerated as the number of counts of asexual parasites per 200 leukocytes, assuming a median leukocyte count of $8000 / \mu \mathrm{L}$. Thin smears 


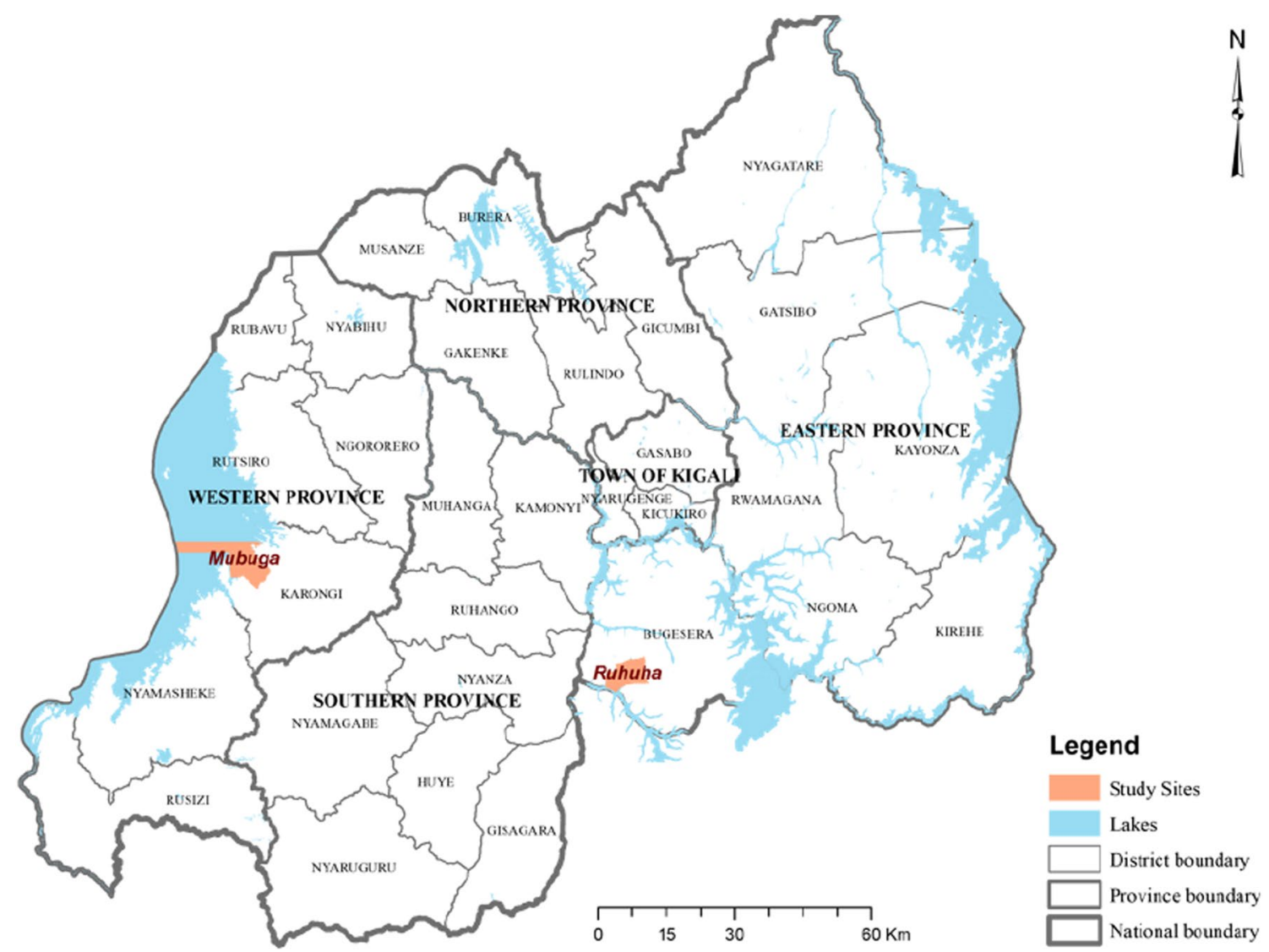

Fig. 1 Location map showing study sites of Ruhuha" and Mubuga" sectors in Rwanda. "Ruhuha sector is located in Bugesera District, Eastern Rwanda whilst Mubuga sectors is located in Karongi District, Western Rwanda

were used to differentiate Plasmodium species. External quality control was done on a $10 \%$ sample of randomly selected thick and thin smears by microscopists at the National Reference Laboratory, Kigali, Rwanda whose results were in agreement with those reported by the study technicians.

\section{Plasmodium falciparum DNA extraction and $m s p-2$ allelic typing}

DNA was extracted with Chelex 100 Resin (Bio-Rad Laboratories, Hercules, CA, USA) as previously described [23]. The surface antigen loci $m s p-2$ was amplified using previously described primers [24]. Briefly, $2 \mu \mathrm{L}$ of template DNA was amplified using nested polymerase chain reaction (nPCR), with second-round primers specific to $m s p-2$ allelic families. PCR products were then separated on a $2.5 \%$ agarose gel (UltraPure Agarose; Invitrogen, Carlsbad, CA, USA) and stained with ethidium bromide. GelCompar II software (Applied Maths, Sint-MartensLatem, Belgium) was used to select alleles and estimate PCR product size as described elsewhere [21].

\section{Statistical analysis}

Demographics, clinical features and bed net use data were collected using hard copy study case record forms while laboratory results were transcribed into study laboratory registers. Both datasets were double-entered into EPI Info ${ }^{\mathrm{TM}} 7$ (Centres for Disease Control and Prevention, GA, USA) database and later transferred into STATA (version 13.1, College Station, TX, USA) for analysis. Parasitaemia - the number of parasites/ $\mu \mathrm{L}$ was graded as low $(<1000)$, moderate $(1000-9999)$ and high $(>10,000)$ as per WHO parasitaemia cut-off for severe malaria in low transmission settings [25]. MOI was defined as the proportion of people who carry more than one allele (genotype) for any of the examined genes. Mean multiplicity of infection (MOI) was estimated by dividing the total number of distinct $m s p 2$ genotypes detected by the number of positive samples.

Descriptive statistics of proportions and means were used to summarize distributions of allelic families, baseline demographics, MOI, and other covariate data. Chi square tests were used to compare mean MOI and allelic variant 
distributions between study sites. Independent t test was used to compare mean MOI outcome by independent factors of age group, study site, history of fever and presence of measured fever $\left(\geq 37.5^{\circ} \mathrm{C}\right)$, sex and bed net use. Expected heterozygosity index (HE) - which measures locus diversity-was calculated using the formulae $\mathrm{HE}=[\mathrm{n} /(\mathrm{n}-1)]$ $\left[\left(1-! P_{i}^{2}\right)\right]$, where $n=$ sample size, $\mathrm{Pi}=$ allelic frequency. Odds ratios (ORs) and $95 \%$ confidence intervals (CIs) were calculated to evaluate the strengths of associations. Statistical significance was defined as $\mathrm{p}$ value $\leq 0.05$ [22].

\section{Ethical clearance}

All adults and carers of children $<18$ years old were informed of the purpose and procedures of the study, and recruited only after obtaining informed written consent. The study was approved by the National Health Research Committee and the Rwanda National Ethics Committee (No. 20/RNEC/2015), Kigali, Rwanda.

\section{Results}

\section{Baseline study participant demographics}

A total of 407 patients who were microscopically confirmed with malaria by health facility laboratory technicians were enrolled. Of these, 402 (98.8 \%) were microscopically re-confirmed by study-trained technicians to be malaria positive. Of the 402, final data analysis was performed on 388 (96.5\%) who were successfully genotyped for the $m s p-2$ alleles. Stratified by site, 195 (50.3\%) cases were enrolled at Ruhuha whilst 193 (49.7\%) enrolled at Mubuga (Table 1). A higher proportion (55.4\%) of study participants were females. The overall group mean age was 15.5 years $(\mathrm{SD} \pm 13.6)$. Overall geometric mean parasite density was 1119.3 parasites/ $\mu \mathrm{L}$.

\section{Demographics, clinical features, parasitological and malaria control use distributions among participants from the two study sites}

The results of group comparisons of demographic, bed net use, fever experiences, and parasite density among patients from the two study sites are shown in Table 1. Significant differences in proportions of participant characteristics of sex $(p=0.04)$ and age group $(p=<0.0001)$ between patients from Ruhuha and Mubuga sites were noted. At Ruhuha, a higher proportion (60.5\%) of patients were females compared to Mubuga (50.3\%). Among children aged $<5$ years, a higher proportion was seen at Ruhuha (26.7 \%) compared to Mubuga (11.4 \%) while among those aged $>15$ years, a higher proportion was enrolled at Mubuga (40.4\%) compared to Ruhuha (24.6\%). With regard to history of reported fever, a significantly ( $\mathrm{p}=0.001)$ higher proportion $(99 \%)$ was noted at Ruhuha compared to that reported at Mubuga (84 \%). In contrast, a significantly higher proportion of patients $(\mathrm{p}=0.001)$ with a measured temperature of $\geq 37.5{ }^{\circ} \mathrm{C}$ were seen at Mubuga (58.5\%) compared to that reported from Ruhuha (41.5\%). A significantly higher proportion (38.0\%) of patients at Ruhuha had high parasite count $(>10,000$ parasites $/ \mu \mathrm{L})$ than those seen at Mubuga $(3.1 \%$;

Table 1 Demographics, malaria prevention, clinical profiles and geometric mean parasite densities/* $\mu \mathrm{L}$ for malaria cases identified in Ruhuha and Mubuga sites in Rwanda

\begin{tabular}{|c|c|c|c|c|}
\hline \multicolumn{2}{|l|}{ Variables } & \multirow{2}{*}{$\begin{array}{l}\text { Ruhuha site } \mathrm{n}=195 \\
\mathrm{n}(\%)\end{array}$} & \multirow{2}{*}{$\begin{array}{l}\text { Mubuga site } n=193 \\
n(\%)\end{array}$} & \multirow[t]{2}{*}{ Pearson's $\mathrm{X}^{2}$ test } \\
\hline Demographics & Variable sub-groups & & & \\
\hline \multirow[t]{3}{*}{ Age groups } & 6 months -5 years & $52(26.7)$ & $22(11.4)$ & - \\
\hline & $6-15$ years & $95(48.7)$ & $93(48.2)$ & - \\
\hline & 16-73 years & $48(24.6)$ & $78(40.4)$ & $<0.0001$ \\
\hline \multirow[t]{2}{*}{ Sex } & Males & $77(39.5)$ & $96(49.7)$ & - \\
\hline & Females & $118(60.5)$ & $97(50.3)$ & 0.042 \\
\hline Malaria prevention used & $\begin{array}{l}\text { Number reporting bed net use } \\
\text { night prior to survey }\end{array}$ & $129(66.2)$ & $150(77.7)$ & 0.011 \\
\hline \multirow[t]{2}{*}{ Fever history and experience } & $\begin{array}{l}\text { Number with history of fever in } \\
\text { previous } 24 \mathrm{~h}\end{array}$ & $192(98.5)$ & $162(83.9)$ & $<0.0001$ \\
\hline & $\begin{array}{l}\text { Number with tympanic temperature } \\
\text { of } \geq 37.5^{\circ} \mathrm{C}\end{array}$ & $78(41.5)$ & $110(58.5)$ & 0.001 \\
\hline \multirow[t]{4}{*}{ Parasitology } & $\begin{array}{l}\text { Parasite count ranges/per } \mu \mathrm{L} \text { Low } \\
\quad(<1000)\end{array}$ & $63(32.3)$ & $113(58.6)$ & \\
\hline & Moderate (1000-9999) & $58(29.7)$ & $74(38.3)$ & \\
\hline & Severe $(\geq 10,000)$ & $74(38.0)$ & $6(3.1)$ & $<0.0001$ \\
\hline & $\begin{array}{l}\text { Geometric mean parasitaemia } \\
\text { (parasites } / \mu \mathrm{L})\end{array}$ & 2347.3 (95 \% Cl: 1772.1-3109.2) & 529.7 (95 \% Cl: 402.3-697.4) & $<0.0001$ \\
\hline
\end{tabular}


$\mathrm{p}<0.0001)$. Similarly, geometric mean parasitaemia counts were higher at Ruhuha (95 \% CI: 5686.5-7394.8) than at Mubuga (95\% CI: 1383.3-2251.7). Bed net use was significantly higher at Mubuga $(77.7 \%)$ than at Ruhuha (66.2\%) ( $\mathrm{p}=0.001)$.

\section{Infection clones and allelic diversity}

Overall, a range of one to six infection clones per sample was seen. At both sites, about $55.4 \%$ of the infections were monoclonal, with isolates from the Mubuga site carrying a significantly higher proportion of monoclonal infections (73\%) compared to those from Ruhuha (38\%) $(\mathrm{p}<0.0001)$. The numbers of strains per isolate are presented in Table 2. Overall, a total of 80 (27.8 \%) samples

Table 2 Plasmodium falciparum msp-2 PCR product numbers, size by base pair range and $H_{E}$ for isolates with $\geq 0$ one allele identified

\begin{tabular}{|c|c|c|c|}
\hline $\begin{array}{l}\text { Variable charac- } \\
\text { teristic }\end{array}$ & $\begin{array}{l}\text { Variable } \\
\text { sub-group }\end{array}$ & Ruhuha n (\%) & Mubuga n (\%) \\
\hline \multirow{6}{*}{$\begin{array}{l}\text { Number of clones } \\
\text { per sample }\end{array}$} & 1 & $74(38.0)$ & $141(73.0)$ \\
\hline & 2 & $60(30.8)$ & $48(24.9)$ \\
\hline & 3 & 35 (17.9) & $3(1.6)$ \\
\hline & 4 & $18(9.2)$ & $1(0.5)$ \\
\hline & 5 & $3(1.5)$ & $0(0.0)$ \\
\hline & 6 & $5(2.6)$ & 0 \\
\hline \multicolumn{4}{|c|}{$m s p-2$ strain distribution } \\
\hline \multirow[t]{5}{*}{ 3D7 strain ${ }^{a}$} & 0 & $33(16.9)$ & $65(33.7)$ \\
\hline & 1 & $93(47.7)$ & $122(63.2)$ \\
\hline & 2 & $53(27.2)$ & $5(2.6)$ \\
\hline & 3 & $15(7.7)$ & $1(0.3)$ \\
\hline & 4 & $1(0.5)$ & $0(0.0)$ \\
\hline \multirow[t]{5}{*}{ FC27 strain $^{b}$} & 0 & $78(40.0)$ & $86(44.6)$ \\
\hline & 1 & $86(44.1)$ & $99(51.3)$ \\
\hline & 2 & $15(7.7)$ & $8(4.2)$ \\
\hline & 3 & $11(5.6)$ & $0(0.0)$ \\
\hline & 4 & $5(2.6)$ & $0(0.0)$ \\
\hline \multicolumn{4}{|c|}{ PCR products per base pair range } \\
\hline \multirow[t]{6}{*}{ FC27 } & $300-330$ & $95(51.9)$ & $114(85.1)$ \\
\hline & $350-380$ & $58(31.4)$ & $17(12.7)$ \\
\hline & $400-430$ & $15(8.1)$ & $2(1.5)$ \\
\hline & $450-600$ & $16(8.6)$ & $1(0.7)$ \\
\hline & $\begin{array}{l}\text { Total FC27 PCR } \\
\text { products }\end{array}$ & 184 & 134 \\
\hline & $\begin{array}{l}\text { HE average HE } \\
\quad(0.44)\end{array}$ & 0.62 & 0.26 \\
\hline \multirow[t]{4}{*}{ 3D7 } & $200-300$ & $166(55.7)$ & $120(83.3)$ \\
\hline & $320-400$ & $132(44.3)$ & $22(16.7)$ \\
\hline & $\begin{array}{l}\text { Total 3D7 PCR } \\
\text { products }\end{array}$ & 298 & 144 \\
\hline & $\begin{array}{l}\text { HE average } H E \\
\quad(0.39)\end{array}$ & 0.49 & 0.28 \\
\hline
\end{tabular}

\footnotetext{
a 3 D7 strain difference in distribution $=x^{2}<0.0001$
}

b FC27 strain difference in distribution $=x^{2}=0.001$ were co-infected by both FC27 and 3D7 types but with the number of strains per isolate noted to be higher at Ruhuha ( $\mathrm{p}<0.0001$ ) compared to Mubuga (Table 2). In total, more 3D7 allelic variants were detected (298) compared to FC27 variant (184) alleles.

\section{Allelic frequency and heterozygosity}

For both FC27 and 3D7 alleles, 760 distinct P. falciparum clones were detected (Table 2). Parasite allelic frequency varied among isolates from the two study sites (Figs. 2, 3). Overall, the majority (68 \%) of isolates carried the FC27 300-330-bp size fragment (Fig. 3) while $70 \%$ carried the 3D7 200-300-bp size fragment (Fig. 2). At Ruhuha, HE for 3D7 and FC27 was 0.49 and 0.62 while at Mubuga, HE for 3D7 and FC27 was 0.28 and 0.26 , respectively (Table 2). At each of the 3D7 (Fig. 2) and FC27 (Fig. 3) alleles, higher levels of polymorphisms were seen among isolates from Ruhuha than isolates from Mubuga.

\section{Multiplicity of infection}

Results for determinants of MOI are shown in Table 3. Overall, MOI for all infections at both sites was $\sim 1.7$. However, MOI varied significantly $(p<0.0001)$ between Mubuga (1.3) and Ruhuha (2.1). In this study, MOI was seen to increase proportional to age group being from 1.7 among those under 5 years old to 1.9 among those aged $6-15$ years and 1.5 among those $>15$ years. Isolates from Ruhuha also had higher MOI compared to those from Mubuga.

\section{Discussion}

This study reports, for the first time in Rwanda, a differential spatial distribution of patient demographics of age and sex, fever, parasite density and P. falciparum genetic diversity across two study sites. A higher geometrical mean parasite counts (2347 vs 530 parasites), more polyclonal infections, higher MOI and higher allelic frequency were observed at higher malaria-endemic Ruhuha compared to the lower malaria-endemic Mubuga area.

A higher proportion of children aged $<5$ years was enrolled at Ruhuha compared to Mubuga while, in contrast, a higher proportion of those aged $>15$ years was recruited at Mubuga compared to Ruhuha. Higher malaria burden in younger age groups in settings of high malaria transmission intensity have been reported previously [26-28]. The age-related association of disease severity across different malaria transmission zones is currently poorly elucidated particularly in the era of scaled-up interventions such as LLINs and IRS whose impact on reducing malaria transmission has also influenced age-related malaria risk. As reported elsewhere 


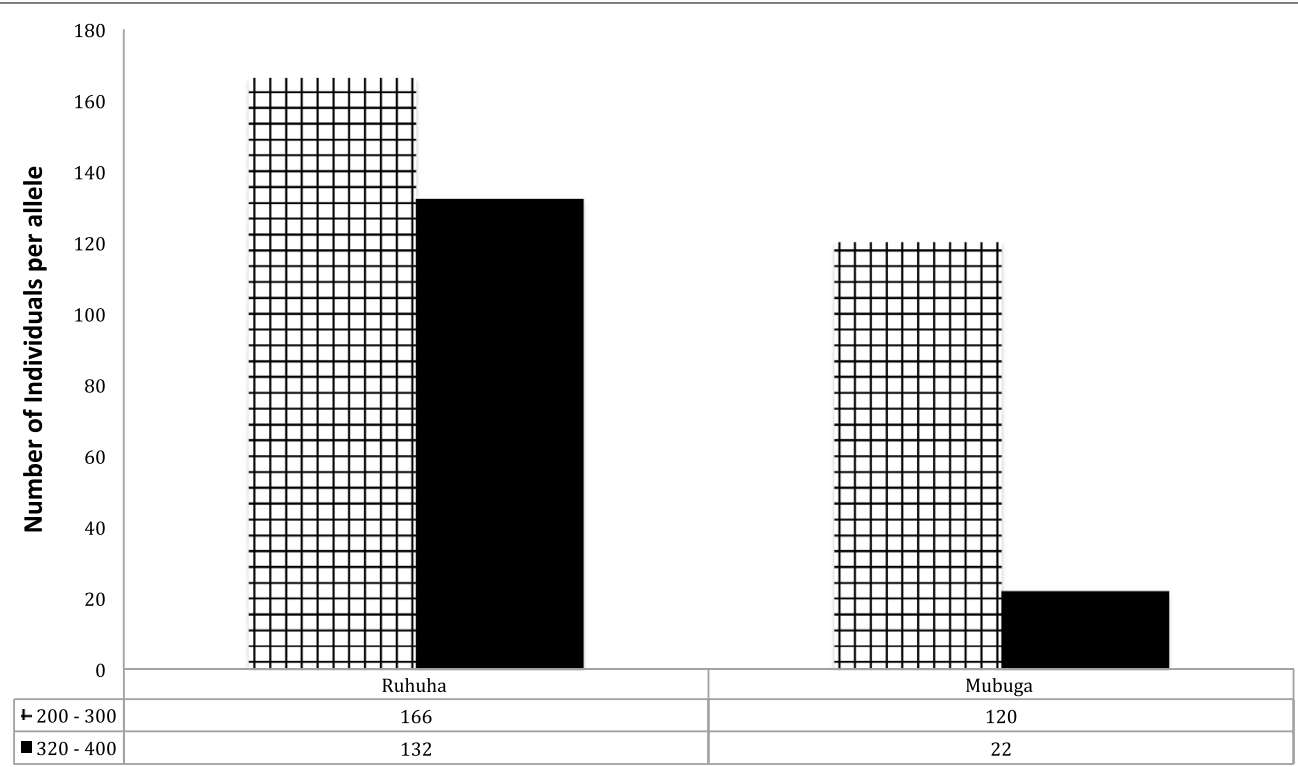

Fig. 2 Distribution of msp-2 3D7 alleles across Ruhuha and Mubuga study sites in Rwanda

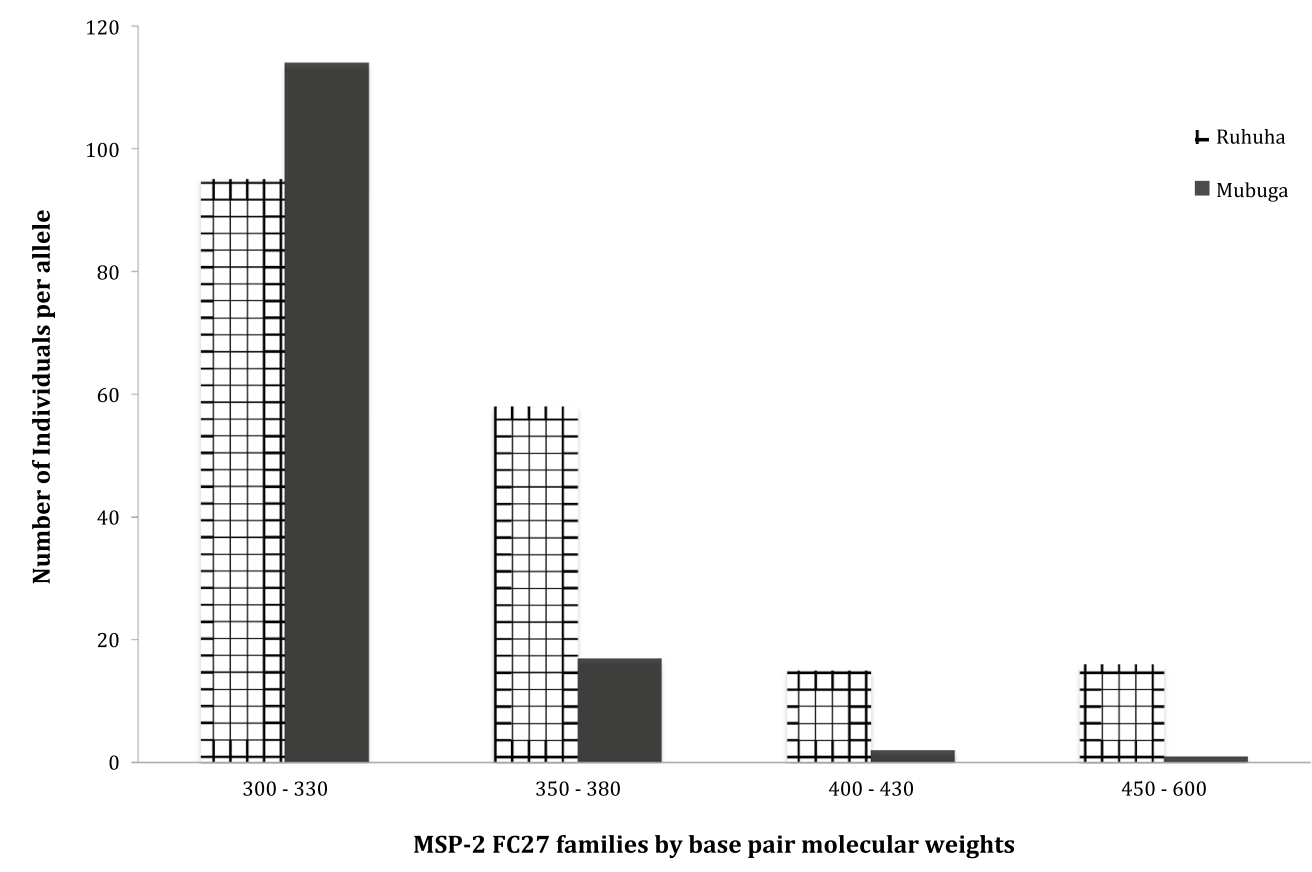

Fig. 3 Distribution of msp-2 FC27 alleles between Ruhuha and Mubuga study sites, Rwanda

scale-up of LLINs has been done [29-32], this study provides further evidence of a shift towards higher malaria risk in older age groups. Results from this study may be confounded by the age-distribution differences between the two sites, with the higher malaria-endemic Ruhuha sector having a higher proportion of sick children aged $<5$ years. A higher risk of $P$. falciparum infection among younger age groups has been reported from elsewhere, particularly for severe malaria [33]. The apparent higher risk of malaria among younger age groups at the higher endemic Ruhuha site was probably due to a lower clinical protective immunity among the younger age group ( $<5$ years) relative to older age groups $(6-15$ and $>15$ years) who may have a higher degree of partially 
Table 3 Bivariate analysis for covariate determinants of multiplicity of infection (MOI)

\begin{tabular}{|c|c|c|c|c|c|c|}
\hline Variable & Variable sub-group & n (\%) & $\begin{array}{l}\text { M01 Ruhuha, } \\
\mathrm{n}=195\end{array}$ & $\begin{array}{l}\text { MOI Mubuga, } \\
n=193\end{array}$ & $\begin{array}{l}\text { Overall MOI, } \\
( \pm S D)\end{array}$ & $p$ value \\
\hline Study site & All & $388(100 \%)$ & 2.13 & 1.29 & $1.72( \pm 1.02)$ & $<0.0001$ \\
\hline \multirow{3}{*}{$\begin{array}{l}\text { Study participants } \\
\text { age group }\end{array}$} & $\leq 5$ years & $74(19.1)$ & 1.92 & 1.32 & $1.74( \pm 1.05)$ & \\
\hline & $6-15$ years & $188(48.4)$ & 2.34 & 1.37 & $1.86( \pm 1.07)$ & \\
\hline & $\geq 16$ years & $126(32.5)$ & 1.96 & 1.21 & $1.49( \pm 0.89)$ & 0.008 \\
\hline \multirow[t]{2}{*}{ Sex } & Male & $173(44.6)$ & 2.14 & 1.27 & $1.66( \pm 0.97)$ & \\
\hline & Female & $215(55.4)$ & 2.13 & 1.32 & $1.76( \pm 1.06)$ & 0.321 \\
\hline \multirow{2}{*}{ Measured fever $\geq 37.5^{\circ} \mathrm{C}$} & Yes & $188(48.4)$ & 2.12 & 1.29 & $1.79( \pm 1.10)$ & \\
\hline & No & $200(51.6)$ & 2.15 & 1.30 & $1.63( \pm 0.92)$ & 0.119 \\
\hline \multirow[t]{2}{*}{ Reported fever } & Yes & 354 (91.2) & 2.14 & 1.29 & $1.75( \pm 1.05)$ & \\
\hline & No & $34(8.8)$ & 2.00 & 1.32 & $1.38( \pm 0.65)$ & 0.046 \\
\hline \multirow{3}{*}{ 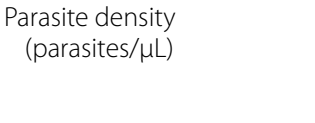 } & $<1000$ & $176(45.4)$ & 1.91 & 1.28 & $1.51( \pm 0.82)$ & \\
\hline & 1000-9999 & $132(34.0)$ & 2.39 & 1.31 & $1.79( \pm 1.10)$ & \\
\hline & $\geq 10,000$ & $80(20.6)$ & 2.12 & 1.33 & $2.06( \pm 1.18)$ & 0.0002 \\
\hline \multirow{2}{*}{$\begin{array}{l}\text { Number of Plasmodium } \\
\text { species }\end{array}$} & P. falciparum only & $215(55.4)$ & 2.33 & 1.11 & $1.73( \pm 1.03)$ & \\
\hline & P. falciparum and P. ovale) & $173(44.6)$ & 2.13 & 1.30 & $1.42( \pm 0.67)$ & 0.303 \\
\hline \multirow[t]{2}{*}{ Presence of gametocyte } & Yes & $10(2.8)$ & 1.50 & 1.38 & $1.30( \pm 0.56)$ & \\
\hline & No & $378(97.2)$ & 2.14 & 1.29 & $1.72( \pm 1.03)$ & 0.322 \\
\hline \multirow{2}{*}{$\begin{array}{l}\text { History of sleeping under a } \\
\text { bed net the night before } \\
\text { survey }\end{array}$} & Yes & $279(71.9)$ & 2.21 & 1.28 & $1.71( \pm 1.09)$ & \\
\hline & No & $109(28.1)$ & 1.99 & 1.35 & $1.73( \pm 0.84)$ & 0.834 \\
\hline
\end{tabular}

protective immunity already in high transmission settings. In contrast, where malaria control activities, particularly LLIN usage, were scaled up, malaria risk has been observed to shift to older age groups for reasons including delays in acquiring immunity and less bed net use among the older age groups of 6-15 years, compared to children $<5$ years. A spatial and temporal analysis of changing transmission intensities may provide clarity on allelic frequency epidemiology as determinants of setting-specific malaria risk.

Among patients enrolled at Ruhuha, a significantly higher proportion were females in contrast to those recruited at Mubuga where both sexes were proportionally represented. The association between malaria risk and sex remains equivocal. In contrast to this study's findings, at the Ruhuha site, a number of previous studies, including two conducted at the Ruhuha site, reported a bias towards higher malaria risk among males [31, 32, 34, 35]. The observed higher proportion of females at Ruhuha in this study may be a chance occurrence due to the non-randomized study design used. In addition, females, as seen in Rwanda, tend to have better health-seeking behaviour, including more frequent visits to health facilities and are more likely to be recruited in health system-based studies than their male counterparts. This is the most probable reason for findings reported here, particularly given that it has been previously established that males had a higher malaria risk in Ruhuha compared to females [31, 34].

In this study, the proportion of patients with a reported fever experiences and by a fever $\geq 37.5^{\circ} \mathrm{C}$ differed across the two sites. Whilst a higher proportion of Ruhuharecruited patients self-reported a history of fever in the last $24 \mathrm{~h}$ compared to those from Mubuga and, in contrast, a lower proportion of the same patients from Ruhuha were confirmed with a measured fever (tympanic temperature $\geq 37.5{ }^{\circ} \mathrm{C}$ ) compared to Mubuga patients. Fever is a common malaria-associated symptom and a major reason for seeking care among suspected malaria in endemic settings. At the higher malaria-endemic Ruhuha site, it is plausible that residents are more likely to associate fever with malaria and hence the higher proportion of reported fevers. On the other hand, at the lower malaria-endemic Mubuga site, with presumably a lower proportion of individuals with partially protective levels of immunity, patients are more likely to have symptomatic malaria infections presenting with fever than those at Ruhuha. However, the higher proportion of children $<5$ years old in Ruhuha may have confounded the observed higher proportion of reported fevers in Ruhuha compared to Mubuga with malaria being associated with fever or recent history of fever in infants. In contrast, the higher malaria endemicity in Ruhuha may plausibly be associated with higher levels of protective 
immunity leading to a lower proportion of malaria compared to persons from the lower-endemic Mubuga site, as previously reported from the Ruhuha site [31, 34]. Characterizing the association between fever experiences and malaria risk is complicated by other determinants of measured fevers, including population access to and use of antipyretic medications prior to visiting a health facility.

In this study, mean MOI was significantly higher at the higher malaria-endemic Ruhuha site compared to the lower malaria-endemic Mubuga site. While many studies have reported comparable findings of higher MOI in higher endemic settings and correspondingly lower MOI in low endemic settings [17, 20,36,37], a study in Ghana did not find any association between MOI and transmission intensity [38]. A plausible reason for higher MOI in higher endemic settings may be the greater diversity and the more frequent meiotic recombination in higher malaria transmission settings. In this study, MOI was noted to significantly decrease with increasing age. Previous studies on associations between MOI and age groups have shown mixed findings, with some reporting no association $[36,39,40]$, while others have reported comparable findings of lower MOI with increasing age have been demonstrated in Nigeria, Ghana and Senegal as seen in this study [11, 38, 41]. In a Tanzanian study among children, MOI peaked among those aged 3-7 years suggesting that younger age groups ( $<10$ years) may be contributing significantly to driving parasite diversity [42]. A possible reason for the conflicting findings to those in this study may include differences in study age groups and study site malaria intensities. It is plausible that multiple strains are needed to develop immunity in younger children and hence the higher diversity in younger children. Contrastingly, pre-existing immunity in older age groups may be selectively clearing out some strain types and hence the noted inverse association between MOI and age.

In this study, MOI was positively correlated with parasite density. This finding accords with previous studies where higher MOI among individuals with higher parasite densities has been demonstrated [11, 43]. In contrast, no association between MOI and level of parasitaemia was reported elsewhere [36]. Because parasite densities are influenced by multiple determinants including age, levels of exposure to malaria infections and area-specific transmission levels, these latter factors may partially-either individually or collectively-account for the lack of MOI and parasitaemia level associations observed elsewhere.

About $55 \%$ of the P. falciparum msp- 2 confirmed isolates carried monoclonal (single allele) infection. By study site, a higher proportion of monoclonal infections were seen at Mubuga (73.1\%) compared to Ruhuha (38.0 \%).
These data are similar to other studies where higher proportions of $>50 \%$ and up to $100 \%$ polyclonal infections have been seen in meso-endemic and holo-endemic settings [35, 44, 45]. Similarly, based on $m s p-1$ genetic diversity marker, higher proportions of polyclonal infection have been seen in high endemicity settings, suggesting that malaria parasite polyclonality may be a useful proxy measure of level of endemicity [46]. Overall, genetic diversity was higher at the more malaria-endemic Ruhuha site than at Mubuga whilst 3D7 allelic families were more frequent than the FC27 families. At Ruhuha, 3D7 PCR products were 1.6-fold more than FC27 PCR products. Based on $m s p-1$, similar observations of higher diversity at a holo-endemic site in Tanzania compared to hypo-endemic south-western Brazilian Amazon and meso-endemic southern Vietnam has been reported, with 3D7 reported as the most frequently circulating allele in this study [47].

The majority of msp-2 FC27 alleles belonged to the 300-330-bp allele family while the most prevalent $m s p$ 2 3D7 allele belonged to the 200-300-bp allele family. Between the two sites, while the 300-330-bp allele was more frequent at Mubuga, the larger size (350-380, 400-450, 480-600) allelic families were more common at Ruhuha. In contrast to the FC27 gene, the 200-330bp allele was the most frequent circulating allele at both Ruhuha and Mubuga. Of interest, findings from Mubuga of lower allelic diversity and lower frequency of circulating alleles point to a high likelihood of re-infection with the same allele. Differentiating between recrudescence and re-infection using $m s p$ - 2 in a low-endemic setting such as Mubuga may be limited by the $m s p-2$ low discriminatory power.

A number of factors, including an adequate sample size, use of validated genetic marker for diversity and allelic frequency, use of an automated gel reader to determine allelic family base pair sizes and a comparative analysis for the two study groups drawn from settings of different malaria transmission intensities, are major strengths of this study. However, there were some limitations. Firstly, there was a lack of earlier data on transmission intensity at either study sites to delineate local malaria endemicities. Secondly, being a cross-sectional survey design, study findings can only provide a baseline comparator for current diversity and disease clinical profiles but is unable to determine the value of diversity on other disease outcomes other than parasite density as well as time and impact of used intervention related effects. Thirdly, the study was done at two sites whilst in Rwanda, malaria risk is categorized into four malaria ecologic zones. Therefore, study findings may have limited generalizability, restricted to settings of comparable transmission and malaria control tool implementation levels. Fourthly, due 
to cost restrictions, we used a valid but lower discriminatory power assay (agarose gel electrophoresis) compared to other assays (e.g. capillary electrophoresis) and thus findings may be of a lower accuracy. Lastly, although $m s p-2$ is a validated molecular marker of diversity, use of one marker may miss variations at other polymorphic loci and underestimate the real magnitude of diversity.

\section{Conclusion}

This study demonstrated a differential distribution in demographics, measured temperature, $P$. falciparum parasite density and genetic diversity as well as allelic distribution between individuals from two sites of variable malaria transmission intensities. HE and mean MOI were higher among isolates collected from the higher malaria Ruhuha site. Locally, characterizing malaria disease severity, based on clinical features and parasitaemia levels, across populations from settings of differing malaria transmission intensities is important in profiling malaria risk maps and in decision-making on which control tools may have optimal impact. The difference in diversity may have differential effects on multiple parameters including drug-resistant profiles, immunological responses to anti-malarial drug and effectiveness of vaccines tested in Rwanda in the future.

\section{Authors' contributions}

FK conceived the idea, designed the study, participated in performance of the experiments, analysed the data and drafted the manuscript. SLN participated in performing the experiments and revised the manuscript substantially. ST performed the experiments and provided in put in writing the manuscript. EH, PFM and MPG provided substantial contribution to the manuscript writing. KN supported study protocol development and provided substantial input in the writing of the manuscript. MvV was involved in the conception of the study, supported field implementation work and participated in the writing of manuscript. All authors read and approved the final manuscript.

\section{Author details \\ ${ }^{1}$ Medical Research Centre Division, Rwanda Biomedical Centre, PO Box 7162, Kigali, Rwanda. ${ }^{2}$ Division of Internal Medicine, Department of Infectious Diseases, Centre of Tropical Medicine and Travel Medicine, Meibergdreef 9, 1100 DD Amsterdam, The Netherlands. ${ }^{3}$ Molecular Research Laboratory, Infectious Disease Research Collaboration, New Mulago Hospital Complex, PO Box 7051, Kampala, Uganda. ${ }^{4}$ Department of Pathology, School Biomedical Science, College of Health Science, Makerere University, PO Box 7072, Kam- pala, Uganda. ${ }^{5}$ Royal Tropical Institute/Koninklijk Instituutvoor de Tropen, KIT Biomedical Research, Meibergdreef 39, 1105 AZ Amsterdam, The Netherlands. ${ }^{6}$ School of Medicine, College of Medicine and Health Sciences, University of Rwanda, PO Box 3286, Kigali, Rwanda. ${ }^{7}$ Department of Tropical Medicine, School of Public Health and Tropical Medicine, Vector-Borne Infectious Disease Research Centre, Tulane University, 333 S Liberty Street, Mail code 8317, New Orleans, LA 70112, USA.}

\section{Acknowledgements}

We thank study participants, their parents and/or guardians as well as health facility leadership and personnel for participating and supporting conduct of the study. This study was financially supported by the NIH Fogarty International Centre through Grant \#5R25TW009340 to FK as part of his Fogarty Global Health Fellowship. Supplementary financial support for sample analysis was received from the Netherlands Organization for Tropical Scientific Research (NWO-WOTRO through Grant \# SA358001 to the Academic Medical Centre, University of Amsterdam, The Netherlands.

\section{Competing interests}

The authors declare that they have no competing interests.

Received: 6 January 2016 Accepted: 13 April 2016

Published online: 26 April 2016

References

1. WHO. World Malaria Report 2014. Geneva: World Health Organization; 2014. http://www.who.int/malaria/publications/world_malaria_ report_2014/wmr-2014-no-profiles.pdf. Accessed 12 May 2015.

2. Steketee R, Campbell C. Impact of national malaria control scale-up programmes in Africa: magnitude and attribution of effects. Malar J. 2010;9:299.

3. Otten M, Aregawi M, Were W, Karema C, Medin A, Bekele W, et al. Initial evidence of reduction of malaria cases and deaths in Rwanda and Ethiopia due to rapid scale-up of malaria prevention and treatment. Malar J. 2009;8:14.

4. Maitland K, Marsh K. Pathophysiology of severe malaria in children. Acta Trop. 2004;90:131-40.

5. USAID. Presidential Malaria Initiative-Rwanda, Malaria Operational Plan FY 2015. 2015. http://www.pmi.gov/docs/default-source/default-document-library/malaria-operational-plans/fy-15/fy-2015-rwanda-malariaoperational-plan.pdf?sfvrsn=3. Accessed 13 Jan 2016.

6. Karema C, Aregawi MW, Rukundo A, Kabayiza A, Mulindahabi M, Fall IS, et al. Trends in malaria cases, hospital admissions and deaths following scale-up of anti-malarial interventions, 2000-2010 Rwanda. Malar J. 2012;11:236.

7. Healer J, Murphy V, Hodder AN, Masciantonio R, Gemmill AW, Anders RF, et al. Allelic polymorphisms in apical membrane antigen-1 are responsible for evasion of antibody-mediated inhibition in Plasmodium falciparum. Mol Microbiol. 2004;52:159-68.

8. Ofosu-Okyere A, Mackinnon MJ, Sowa MP, Koram KA, Nkrumah F, Osei YD, et al. Novel Plasmodium falciparum clones and rising clone multiplicities are associated with the increase in malaria morbidity in Ghanaian children during the transition into the high transmission season. Parasitology. 2001;123:113-23.

9. Sakihama N, Ohmae H, Bakote'e B, Kawabata M, Hirayama K, Tanabe K. Limited allelic diversity of Plasmodium falciparum merozoite surface protein 1 gene from populations in the Solomon Islands. Am J Trop Med Hyg. 2006;74:31-40.

10. Onway DJ, Cavanagh DR, Tanabe K, Roper C, Mikes ZS, Sakihama N, et al. A principal target of human immunity to malaria identified by molecular population genetic and immunological analyses. Nat Med. 2000;6:689-92.

11. Engelbrecht F, Togel E, Beck HP, Enwezor F, Oettli A, Felger I. Analysis of Plasmodium falciparum infections in a village community in Northern Nigeria: determination of MSP-2 genotypes and parasite-specific lgG responses. Acta Trop. 2000;74:63-71.

12. Owusu-Agyei S, Smith T, Beck HP, Amenga-Etego L, Felger I. Molecular epidemiology of Plasmodium falciparum infections among asymptomatic inhabitants of a holoendemic malarious area in northern Ghana. Trop Med Int Health. 2002;7:421-8.

13. Mobegi VA, Loua KM, Ahouidi AD, Satoguina J, Nwakanma DC, Amambua-Ngwa A, et al. Population genetic structure of Plasmodium falciparum across a region of diverse endemicity in West Africa. Malar J. 2012;11:230.

14. Babiker HA, Charlwood JD, Smith T, Walliker D. Gene flow and crossmating in Plasmodium falciparum in households in a Tanzanian village. Parasitology. 1995;111:433-42.

15. Chaitarra V, Holm I, Bentley GA, Petres S, Longacre S. The crystal structure of $C$-terminal merozoite surface protein 1 at 1.8 resolution, a high protective malaria vaccine candidate. Mol Cell. 1999;3:457-64.

16. Ferreira MU, Hartl DL. Plasmodium falciparum: worldwide sequence diversity and evolution of the malaria vaccine candidate merozoite surface protein-2 (MSP-2). Exp Parasitol. 2007;115:32-40.

17. Haddad D, Snounou G, Mattei D, Enamorado IG, Figueroa J, Stahl S, et al. Limited genetic diversity of Plasmodium falciparum in field isolates from Honduras. Am J Trop Med Hyg. 1999;60:30-4. 
18. Babiker HA, Lines J, Hill WG, Walliker D. Population structure of Plasmodium falciparum in villages with different malaria endemicity in east Africa. Am J Trop Med Hyg. 1997;56:141-7.

19. Peyerl-Hoffmann G, JelinekT, Kilian A, Kabagambe G, Metzger WG, von Sonnenburg F. Genetic diversity of Plasmodium falciparum and its relationship to parasite density in an area with different malaria endemicities in West Uganda. Trop Med Int Health. 2001;6:607-13.

20. Babiker H, Ranford-Cartwirht LC, Walliker D. Genetic structure and dynamics of Plasmodium falciparum infection in the kilombero region of Tanzania. Trans R Soc Med Hyg. 1999;93:11-4.

21. Cattamanchi A, Kyabayinze D, Hubbard A, Rosenthal PJ, Dorsey G. Distinguishing recrudescence from reinfection in a longitudinal antimalarial drug efficacy study: comparison of results based on genotyping of msp1, msp-2, and glurp. Am J Trop Med Hyg. 2003;68:133-9.

22. Mwingira F, Nkwengulila G, Schoepflin S, Sumari D, Beck H-P, Snounou G, et al. Plasmodium falciparum msp1, msp2 and glurp allele frequency and diversity in sub-Saharan Africa. Malar J. 2011;10:79.

23. Plowe CV, Djimde A, Bouare M, Doumbo O, Wellems TE. Pyrimethamine and proguanil resistance-conferring mutations in Plasmodium falciparum dihydrofolate reductase: polymerase chain reaction methods for surveillance in Africa. Am J Trop Med Hyg. 1995;52:565-8.

24. Zwetyenga J, Rogier C, Tall A, Fontenille D, Snounou G, Trape JF, et al. No influence of age on infection complexity and allelic distribution in Plasmodium falciparum infections in Ndiop, a Senegalese village with seasonal, mesoendemic malaria. Am J Trop Med Hyg. 1998;59:726-35.

25. WHO. Guidelines for the treatment of malaria. 2nd ed. Geneva: World Health Organization; 2010.

26. Snow RW, Bastos de Azevedo I, Lowe BS, Kabiru EW, Nevill CG, Mwankusye S, et al. Severe childhood malaria in two areas of markedly different falciparum transmission in east Africa. Acta Trop. 1994;57:289-300.

27. Snow RW, Omumbo JA, Lowe B, Molyneux CS, Obiero JO, Palmer A, et al. Relation between severe malaria morbidity in children and level of Plasmodium falciparum transmission in Africa. Lancet. 1997;349:1650-4.

28. Carneiro I, Roca-Feltrer A, Griffin JT, Smith L, Tanner M, Schellenberg JM, et al. Age-patterns of malaria vary with severity, transmission intensity and seasonality in sub-Saharan Africa: a systematic review and pooled analysis. PLoS One. 2010;5:e8988.

29. Akhwale WS, Lum JK, Kaneko A, Eto H, Obonyo C, Björkman A, et al. Anemia and malaria at different altitudes in the western highlands of Kenya. Acta Trop. 2004;91:167-75.

30. Sintasath D, Ghebremeskel T, Lynch M, Kleinau E, Bretas G, Shililu J, et al. Malaria prevalence and associated risk factors in Eritrea. Am J Trop Med Hyg. 2005;72:682-7.

31. Winskill P, Rowland M, Mtove G, Malima RC, Kirby MJ. Malaria risk factors in north-east Tanzania. Malar J. 2011;10:98.

32. Kateera F, Mens PF, Hakizimana E, Ingabire CM, Muragijemariya L, Karinda $P$, et al. Malaria parasite carriage and risk determinants in a rural population: a malariometric survey in Rwanda. Malar J. 2015;14:16.

33. Ceesay SJ, Koivogui L, Nahum A, Taal MA, Okebe J, Affara M, et al. Malaria prevalence among young infants in different transmission settings, Africa. Emerg Infect Dis. 2015;21:1114-21.
34. Rulisa S, Kateera F, Bizimana JP, Agaba S, Dukuzumuremyi J, Baas L, et al. Malaria prevalence, spatial clustering and risk factors in a low endemic area of Eastern Rwanda: a cross sectional study. PLoS One. 2013;8:e69443.

35. Pathak S, Rege M, Gogtay NJ, Aigal U, Sharma SK, Valecha N, et al. Agedependent sex bias in clinical malarial disease in hypoendemic regions. PLoS One. 2012; 7:e35592.

36. Atroosh WM, Al-Mekhlafi HM, Mahdy M, Saif-Ali R, Al-Mekhlafi AM, Surin J. Genetic diversity of Plasmodium falciparum isolates from Pahang, Malaysia based on MSP-1 and MSP-2 genes. Parasit Vectors. 2011;4:233.

37. Arnot D. Unstable malaria in Sudan: the influence of the dry season. Clone multiplicity of Plasmodium falciparum infections in individuals exposed to variable levels of disease transmission. Trans R Soc Trop Med Hyg. 1998;92:580-5.

38. Agyeman-Budu A, Brown C, Adjei G, Adams M, Dosoo D, Dery D, Wilson $\mathrm{M}$, et al. Trends in multiplicity of Plasmodium falciparum infections among asymptomatic residents in the middle belt of Ghana. Malar J. 2013;12:22.

39. Branch OH, Takala S, Kariuki S, Nahlen BL, Kolczak M, Hawley W, et al. Plasmodium falciparum genotypes, low complexity of infection, and resistance to subsequent malaria in participants in the Asembo Bay Cohort Project. Infect Immun. 2001;69:7783-92.

40. Takala SL, Coulibaly D, Thera MA, Dicko A, Smith DL, Guindo AB, et al. Dynamics of polymorphism in a malaria vaccine antigen at a vaccinetesting site in Mali. PLoS Med. 2007;4:e93.

41. Ntoumi F, Contamin H, Rogier C, Bonnefoy S, Trope JF, Mercereau-Puijalon $\mathrm{O}$. Age dependent carriage of multiple Plasmodium falciparum merozoite surface antigen-2 alleles in asymptomatic malaria infection. Am J Trop Med Hyg. 1995;52:81-8.

42. Smith T, Felger I, Kitua A, Tanner M, Beck HP. Dynamics of multiple Plasmodium falciparum infections in infants in a highly endemic area of Tanzania. Trans R Soc Trop Med Hyg. 1999;93:35-9.

43. Smith T, Beck HP, Kitua A, Mwankusye S, Felger I, Fraser-Hurt N, et al. Age dependence of the multiplicity of Plasmodium falciparum infections and of other malariological indices in an area of high endemicity. Trans R Soc Trop Med Hyg. 1999;93:15-20.

44. Mohammed H, Mindaye T, Belayneh M, Kassa M, Assefa A, Tadesse M, et al. Genetic diversity of Plasmodium falciparum isolates based on msp-1 and msp-2 genes from Kolla-Shele area, Arbaminch Zuria District, southwest Ethiopia. Malar J. 2015;14:73.

45. Legrand E, Volney B, Lavergne A, Tournegros C, Florent L, Accrombessi D, et al. Molecular analysis of two local falciparum malaria outbreaks on the French Guiana coast confirms the msp1 B-K1/varD genotype association with severe malaria. Malar J. 2005;4:26.

46. Babiker HA. Plasmodium falciparum population in the unstable malaria area of eastern Sudan is stable and genetically complex. Trans R Soc Trop Med Hyg. 1998;92:585-9.

47. Ferreira MU, Kaneko O, Kimura M, Liu Q, Kawamoto F, Tanabe K. Allelic diversity at the merozoite surface protein-1 (MSP-1) locus in natural Plasmodium falciparum populations: a brief overview. Mem Inst Oswaldo Cruz. 1998;93:631-8.

\section{Submit your next manuscript to BioMed Central and we will help you at every step:}

- We accept pre-submission inquiries

- Our selector tool helps you to find the most relevant journal

- We provide round the clock customer support

- Convenient online submission

- Thorough peer review

- Inclusion in PubMed and all major indexing services

- Maximum visibility for your research

Submit your manuscript at www.biomedcentral.com/submit 